\title{
The influence of eddy currents on the excitation winding impedance of solid and laminated salient pole synchronous machines
}

\author{
Roberto Felicetti $^{1}$ (D) Curt Johan David Abrahamsson ${ }^{1} \cdot$ Urban Lundin $^{1}$
}

Received: 4 November 2019 / Accepted: 17 June 2020 / Published online: 1 July 2020

(c) The Author(s) 2020

\begin{abstract}
This work investigates the establishment of steady-state eddy currents in solid and laminated salient poles and rotor rim of synchronous machines due to a periodic excitation voltage. It shows that the presence of eddy currents in the rotor magnetic circuit has the double effect of increasing the excitation winding AC-resistance and decreasing its magnetizing ACinductance. According to that a simple analytical model is presented in here which allows a rapid rough estimation of the excitation winding AC-resistance when little information is available about the machine geometry and its electric/magnetic materials properties. The model is then verified by reproducing in frequency the excitation winding AC-resistance and the related power loss measured in two synchronous generators. Finally, the limits of reliability and applicability of the model are discussed. The model has implications for periodic field winding current control and voltage regulation in synchronous machines.
\end{abstract}

Keywords Eddy current $\cdot$ Current control $\cdot$ Iron losses $\cdot$ Magnetizing inductance $\cdot$ Salient pole rotor $\cdot$ Skin effect $\cdot$ Stability Voltage control

\section{Introduction}

The occurrence of currents in a metal body moving across a magnetic field or being crossed by a time-varying magnetic field is a well-known phenomenon [1,2]. Currents induced by that way are mainly responsible for heat generation and electrodynamic force reaction into said body. These effects are sometimes welcomed and pursued in specific applications, such as induction ovens, electromagnetic brakes, metal trash sorters, nondestructive testing devices, just to cite a few. On the contrary, when it comes to electrical machines, the same effects are normally undesired. Eddy currents are therefore avoided or weakened as possible, in order to improve efficiency, reliability and life-time expectation of the electromechanical converters. The well-known specific eddy current loss formula $p_{\mathrm{e}}$ for a conductive laminated magnetic core [3] is

Roberto Felicetti

roberto.felicetti@angstrom.uu.se

1 Division for Electricity, Department of Electrical Engineering, Ångström Lab, Uppsala University, Box 534, 75121 Uppsala, Sweden $p_{\mathrm{e}}=\frac{\pi^{2} \hat{B}^{2} f^{2} d_{1}^{2}}{6 \rho} \sim d_{1}^{2}$,

where $\hat{B}$ is the amplitude of the sinusoidal flux density, $f$ its frequency, $\rho$ the specific resistivity of the conductive material and $d_{1}$ its lamination thickness. By showing that the specific eddy current losses are proportional to the second power of the thickness $d_{1}$, Eq. (1) explains why magnetic circuits are usually finely laminated there, where the flux density is varying in time. However, Eq. (1) is valid when the layer is completely penetrated by the magnetic field or, in other words, when the magnetic reaction of the induced eddy current is negligible in comparison with the exciting magneto-motive force (MMF). In some recent applications of field current control in salient pole synchronous machines (SM), such as the Unbalanced Magnetic Pull Compensation technique [4] and the Synchronous Motor Start by Rotor Polarity Inversion [5], the previous assumption is not valid. As soon as a time-varying MMF is set through the excitation winding, the eddy current arising in the massive conductive rotor parts counteracts almost entirely the establishment of a variable magnetizing flux. This phenomenon points to two current control related aspects, which are very important in the design of combined power-electronics and electrical 
machines systems: (1) the influence of the eddy current-reaction on the magnitude of the winding inductance and (2) the additional heat generated into the rotor by a periodic excitation. Dreyfus [6] has succeeded in describing analytically the proportion between resistive and reactive components of the eddy current in an iron core, by relating reactance and resistance of the eddy current paths to the frequencydependent reduced thickness ${ }^{1}$ of the iron. Nevertheless, in Dreyfus' work, the magnetic flux density does not result from the interaction between the exciting and the induced MMF, since the flux density distribution is a starting point in his analysis. More recently, Nethe [7] and Jäschke et al. [8] have been able to determine the reflected impedance [9] of a winding wound on a ferromagnetic core by making use of very advanced analytical models. Both cited works investigate the subject approached by Dreyfus but from a different perspective. Rather than analyzing the local phase displacement between the eddy current density and the exciting MMF, they explore how the induced losses and magnetic reaction globally affect the excitation winding impedance.

In the present paper, a combination of the approaches of $[6-8]$ is presented. By using some simplifying assumptions, the eddy current density and flux density in the salient pole rotor are first obtained as solutions of a differential problem as in [6]. Said solutions are then related to the main geometric dimensions of the electrical machine as in $[7,8]$, in order to evaluate the AC-resistance and inductance of the excitation winding. Resistance and inductance determined by that way show their dependency on frequency enabling the correlation of their cut-off frequencies with the crosssectional shape of the rotor magnetic circuit and the thickness of its eventually laminated steel. This is relevant for the design of those SM, where the exciting flux needs to be adjusted quickly, sometimes periodically, as shown by the applications presented in $[4,5]$. The model suggested in here can help the designer in targeting the suitable rotor geometry and lamination thickness, which ensure the value of the magnetizing inductance to be higher than a given threshold, up to a needed specified frequency. Furthermore, given a first geometric design of the rotor, the frequency-dependent AC-resistance of the field winding can be determined by said model. The AC field winding resistance can be way higher than its DC value, ${ }^{2}$ mainly as result of the eddy current loss induced into the rotor. This fact has been used in the experimental part of the paper for testing to what extent the modeled frequency-dependent AC-resistance is able to

\footnotetext{
${ }^{1}$ It is the ratio of the lamination thickness to the skin-effect-related penetration depth.

2 This is true even adjusting the DC resistance with the contributions of the bare winding skin and proximity effects [10].
}

fit the AC field winding resistance of two SM, different in rated power and shape.

The simplifying assumptions at the base of the suggested model are presented and discussed in the manuscript. They set the limits for the predictive power of the model itself but help greatly in understanding the reaction mechanism of the eddy currents, which is triggered by the excitation control.

\section{Method}

\subsection{Model assumptions}

The principal assumptions used for deriving the suggested field winding are:

(a) the AC-excitation current is considered sinusoidal.

(b) the magnetic problem is regarded as linear.

(c) the airgap anisotropy is not taken into account.

With reference to point (a), the large time constant of the excitation winding means that high frequency components in the applied stator MMF is effectively removed and the AC-excitation current can be considered sinusoidal.

For the same reason in (b), the reactance is the main factor limiting the current in the field winding at very low frequency already. AC-voltages of few hertz and with the same amplitude of the field winding rated DC-voltage do not manage to force an amplitude-like nominal current into the excitation winding. Moreover, the induced eddy current reaction opposes the exciting MMF by reducing it even more. These combined effects set the magnetic flux in the rotor way below the DC-rated one and far away from the upper knee of the machine magnetization curve. Then, the presence of an airgap in the magnetic circuit lets the lower knee of the magnetization curve disappear.

With reference to the assumption (c), the slot harmonics related eddy current loss in the rotor pole-shoes cannot be sensed in terms of increased AC field winding resistance, which place them outside the field of interest of the present work. The inhomogeneity of the flux density in the poleshoes instead, which depends on the special airgap profile of the SM, has a minor weight on the total rotor eddy current loss for two reasons. First, the higher pole coverage in the pole-shoe than in the pole core and rim makes its general flux density level lower than elsewhere in the rotor. Second, the pole-shoes account for way less volume than the rest of the rotor.

\subsection{Simplified magnetic circuit}

Consider a SM with $2 p$ salient poles having $N$ turns each. The coils of said poles are usually connected in series 


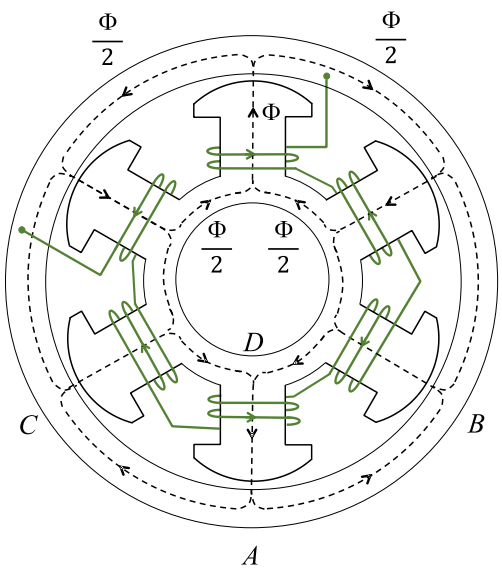

Fig. 1 Linkage between field winding and main flux

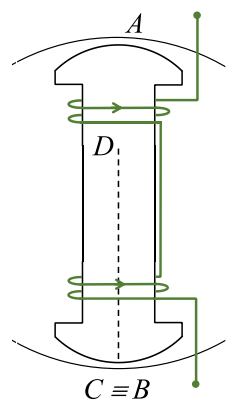

Fig. 2 The elementary electromagnetic cell of the machine

to form one single field winding. The main flux $\Phi$ produced by the excitation winding links the coils as shown in Fig. 1. A series of $p$ circuits with $2 N$-turns can be then recognized, which link all the same flux $\Phi$.

In said figure, the flux is tracked along a path of constant vector potential $A$, from $C$, over $D$ till B.

Figure 2 shows the elementary machine cell obtained by superposing the boundaries $B D$ and $C D$ through an ideal mechanical deformation of the pole-pair structure. It represents $1 / p$ th of the entire machine. Its electrical resistance and inductance are $p$-times smaller than those of the whole field winding.

In order to study the electrical properties of the field winding, it is therefore sufficient to focus on the elementary electromagnetic cell of Fig. 2. Said cell is symmetrical with respect to the vertical and horizontal planes, so that it can be represented in Cartesian coordinates as shown in Fig. 3, after having introduced some approximations:

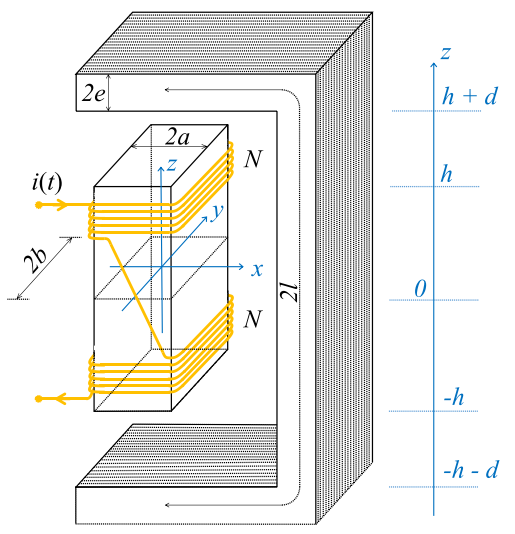

Fig. 3 Cartesian rectangular model of the electromagnetic cell

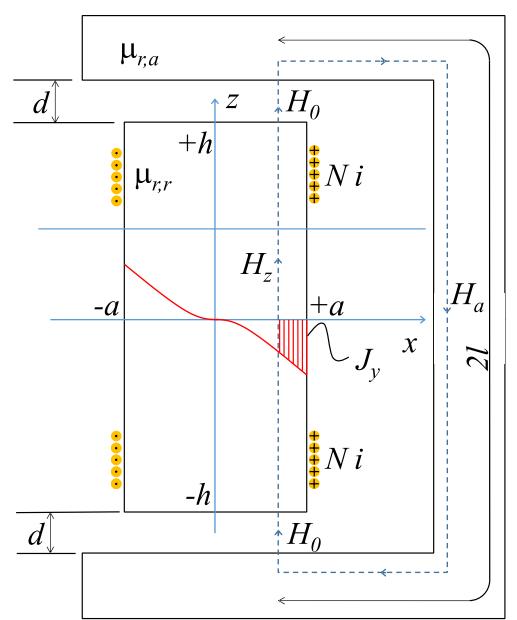

Fig. 4 Application of the Ampère's law along a closed path laying on the $x z$-plane

- the cross section of the rotor rim is assumed to be half of that of the pole core, which is often the case.

- the height of the cell core $2 h$ is equal to the length of the path from $A$ to $C$ through $D$ in Fig. 1.

- the airgap $d$ is the equivalent constant airgap of the machine, providing the same reluctance of the real poleshoe toward the inner stator surface.

- the stator is so finely laminated that the eddy currents affect the rotor only.

\subsection{General solution of the differential problem}

The parallelepiped in Fig. 3 carrying $2 N$ turns of the exciting winding represents a pole-pair of the rotor, including its portion of rim. In the present work, it is referred to it shortly as "core."

Figure 4 represents the application of the Ampère's law along an integration path perpendicular to the $y$-axis. 
It shows that the source of the magnetic field is the MMF provided by the excitation winding.

The linear integration of the magnetic field, along paths that are perpendicular to the $y$-axis and to $x$-axis, respectively, leads to

$\left\{\begin{array}{l}H_{z} \cdot 2 h+H_{0} \cdot 2 d+H_{\mathrm{a}} \cdot 2 l=2 N i+\int_{y_{a}}^{b}-J_{x} 2 h \mathrm{~d} y \\ H_{z} \cdot 2 h+H_{0} \cdot 2 d+H_{\mathrm{a}} \cdot 2 l=2 N i+\int_{x}-J_{y} 2 h \mathrm{~d} x\end{array}\right.$,

where $H_{z}, H_{0}$ and $H_{\mathrm{a}}$ represent the magnetic field in the rotor, in the airgap and in the armature, respectively. The timely varying variable $i$ is the magnetization current, whereas $J_{x}$ and $J_{y}$ are the eddy current density components along the $x$ and $y$-axis, respectively. All magnetic fields are considered constant along their integration paths, which measure $2 h$ in the core, $2 d$ in the airgap and $2 l$ in the armature.

By considering the magnetic flux crossing the airgap to be twice the one flowing in the armature back iron with height $2 e$ (Fig. 3), it is possible to relate $H_{0}$ and $H_{\mathrm{a}}$ by

$H_{\mathrm{a}} \cong \frac{1}{\mu_{r, a}} \frac{a}{e} H_{0}$

where $H_{0}$ is assumed to be constant in the airgap and $\mu_{r, a}$ represents the relative average permeability of the armature back iron. Given (3) Eq. (2) can be rewritten as

$\left\{\begin{array}{l}H_{z} \cdot 2 h+H_{0} \cdot 2 d\left(1+K_{\mathrm{a}}\right)=2 N i+\int_{y}^{b}-J_{x} 2 h \mathrm{~d} y \\ H_{z} \cdot 2 h+H_{0} \cdot 2 d\left(1+K_{\mathrm{a}}\right)=2 N i+\int_{x}-J_{y} 2 h \mathrm{~d} x\end{array}\right.$,

where

$K_{\mathrm{a}}=\frac{l}{\mu_{r, a} d} \frac{a}{e} \cong \frac{l}{\mu_{r, a} d}$

is the magnetic relative length of the armature referred to the airgap. This parameter expresses how many times the magnetic potential drop across the armature is larger than that across the airgap.

In order to determine the eddy currents distribution in the rotor, a differential equation involving magnetic, electric and eddy current density is needed. That equation is

$\nabla^{2} \boldsymbol{H}=\frac{\mu}{\rho} \frac{\partial \boldsymbol{H}}{\partial t}$

which represents the quasi-electrostatic ${ }^{3}$ Helmholtz's equation for the magnetic field $\boldsymbol{H}$ in a conductive isotropic region characterized by uniform resistivity $\rho$ and permeability $\mu$.
Assuming the excitation current to be harmonic with angular frequency $\omega$

$i(t)=I \cos (\omega t)=\operatorname{Re}\left[I \mathrm{e}^{j \omega t}\right]$

and considering that in the core

$\boldsymbol{H}=H_{z} \hat{z}$,

equation (6) can be transformed in

$\frac{\partial^{2} \bar{H}_{z}}{\partial x^{2}}+\frac{\partial^{2} \bar{H}_{z}}{\partial y^{2}}=\bar{\alpha}^{2} \bar{H}_{z}$

The complex parameter $\bar{\alpha}$ in (9) is equal to

$\bar{\alpha}=\frac{1}{\sqrt{2}}(1+j) \sqrt{\frac{\omega \mu}{\rho}}$,

the modulus of which represents the reciprocal of the wellknown penetration depth $\delta$

$\delta=\frac{1}{\|\bar{\alpha}\|}=\sqrt{\frac{\rho}{\omega \mu}}$.

Equation (9) admits a general solution given by [9]

$\bar{H}_{z}=\bar{P}_{0} \cosh \bar{\alpha} y+\sum_{n=1}^{\infty} \bar{Q}_{n} \cosh \sqrt{\bar{\alpha}^{2}+\left(\frac{n \pi}{2 b}\right)^{2}} x \cos \frac{n \pi}{2 b} y$

where

$\bar{P}_{0}=\frac{\bar{H}_{b}}{\cosh (\bar{\alpha} b)}$

and

$\bar{Q}_{n}=\bar{H}_{b} \frac{\frac{\frac{4 \bar{\alpha}^{2}}{n \pi}}{\bar{\alpha}^{2}+\left(\frac{n \pi}{2 b}\right)^{2}}}{\cosh \sqrt{\bar{\alpha}^{2}+\left(\frac{n \pi}{2 b}\right)^{2}} a} \sin \left(n \frac{\pi}{2}\right)$,

with $n=1,3,5 \ldots$ The phasor $\bar{H}_{b}$ in (13) and (14) stands for the value assumed by the magnetic field $\bar{H}_{z}$ on the lateral surface of the iron core.

\subsection{Relating the eddy current and the main flux to the exciting MMF}

Taking the curl of (12), the components of the eddy current density in an arbitrary point of the core can be expressed by

\footnotetext{
${ }^{3}$ In the achievement of (6), the contribution of displacement current density to the curl of $\boldsymbol{H}$ is neglected.
} 


$$
\left\{\begin{array}{l}
\bar{J}_{x}=\bar{\alpha} \bar{P}_{0} \sinh \bar{\alpha} y-\sum_{n=1}^{\infty} \bar{Q}_{n} \frac{n \pi}{2 b} \cosh \sqrt{\bar{\alpha}^{2}+\left(\frac{n \pi}{2 b}\right)^{2}} x \sin \frac{n \pi}{2 b} y \\
\bar{J}_{y}=-\sum_{n=1}^{\infty} \sqrt{\bar{\alpha}^{2}+\left(\frac{n \pi}{2 b}\right)^{2}} \bar{Q}_{n} \sinh \sqrt{\bar{\alpha}^{2}+\left(\frac{n \pi}{2 b}\right)^{2}} x \cos \frac{n \pi}{2 b} y
\end{array} .\right.
$$

However, the coefficients $\bar{P}_{0}$ and $\bar{Q}_{n}$ can only be determined if $\bar{H}_{b}$ is known. To that end, considering the form assumed by (4) on the lateral core surface, the Ampère's law can be formulated as

$H_{0} d\left(1+K_{\mathrm{a}}\right) \mathrm{e}^{j \vartheta_{0}}+H_{b} h \mathrm{e}^{j \vartheta_{b}}=N I$

and represented graphically as shown in Fig. 5.

In Fig. 5, the presence of the eddy current introduces a displacement $\vartheta_{0}$ between the airgap field $\bar{H}_{0}$ and the MMF NI. Since the airgap field $\bar{H}_{0}$ is proportional to the main flux $\bar{\Phi}$, the latter lags on the exciting MMF by the same
However, the main flux $\bar{\Phi}$ can be also determined by using the magnetic field $\bar{H}_{z}$ in the iron core through the following integration

$\bar{\Phi}=\int_{-b}^{b} \int_{-a}^{a} \mu_{0} \mu_{\mathrm{r}} \bar{H}_{b} \mathrm{~d} x \mathrm{~d} y$.

By substituting (13) and (14) in (12), Eq. (18) becomes $\bar{\Phi}=4 a b \mu_{0} \mu_{\mathrm{r}} \bar{H}_{b}\left[\frac{\tanh (\bar{\alpha} b)}{\bar{\alpha} b}+\sum_{n=1}^{\infty} \frac{2\left(\frac{2 \bar{\alpha}}{n \pi}\right)^{2}}{\bar{\alpha}^{2}+\left(\frac{n \pi}{2 b}\right)^{2}} \frac{\tanh \sqrt{\bar{\alpha}^{2}+\left(\frac{n \pi}{2 b}\right)^{2}} a}{\sqrt{\bar{\alpha}^{2}+\left(\frac{n \pi}{2 b}\right)^{2}} a}\right]$.

Assuming $b>a$, (19) can be rewritten as

$\bar{\Phi}=4 a b \mu_{0} \mu_{\mathrm{r}} \bar{H}_{b} \chi \mathrm{e}^{-j \Delta \vartheta}$,

where

$\chi=\left\|\frac{\tanh \left(\frac{1+j}{\sqrt{2}} \frac{b}{\delta}\right)}{\frac{1+j}{\sqrt{2}} \frac{b}{\delta}}+j 2\left(\frac{b}{\delta} \frac{a}{b}\right)^{2} \sum_{n=1}^{\infty}\left(\frac{2}{n \pi}\right)^{2} \frac{\tanh \sqrt{\left(n \frac{\pi}{2} \frac{a}{b}\right)^{2}+j\left(\frac{b}{\delta} \frac{a}{b}\right)^{2}}}{\left[\left(n \frac{\pi}{2} \frac{a}{b}\right)^{2}+j\left(\frac{b}{\delta} \frac{a}{b}\right)^{2}\right]^{3 / 2}}\right\|$

and

$\Delta \vartheta=\left\langle\left(\frac{\tanh \left(\frac{1+j}{\sqrt{2}} \frac{b}{\delta}\right)}{\frac{1+j}{\sqrt{2}} \frac{b}{\delta}}+j 2\left(\frac{b}{\delta} \frac{a}{b}\right)^{2} \sum_{n=1}^{\infty}\left(\frac{2}{n \pi}\right)^{2} \frac{\tanh \sqrt{\left(n \frac{\pi}{2} \frac{a}{b}\right)^{2}+j\left(\frac{b}{\delta} \frac{a}{b}\right)^{2}}}{\left[\left(n \frac{\pi}{2} \frac{a}{b}\right)^{2}+j\left(\frac{b}{\delta} \frac{a}{b}\right)^{2}\right]^{3 / 2}}\right)\right.$.

angle $\vartheta_{0}$, so that

$\bar{\Phi}=4 a b \mu_{0} H_{0} \mathrm{e}^{j \vartheta_{0}}$.
Said $\vartheta_{b}$ the phase of $\bar{H}_{b}$ and $\vartheta_{0}$ the phase of $\bar{H}_{0}$, by equating (17) and (20) the following equations are obtained

$\left\{\begin{array}{l}\chi=\frac{\mu_{0} H_{0}}{\mu_{0} \mu_{\mathrm{r}} H_{b}} \\ \Delta \vartheta=\vartheta_{b}-\vartheta_{0}\end{array}\right.$.

The parameter $\chi$ expresses how much the flux density profile in the core deviates from the DC uniform one, due to the presence of the eddy current. The angle $\Delta \vartheta$ shows instead how much the iron flux density on the lateral surface of the core leads on the airgap flux density. It must be noticed that both terms depend only on the reduced thickness $b / \delta$ and on the core cross-sectional dimensions ratio $a / b$.

In order to find out $\bar{H}_{b}$ and $\bar{H}_{0}$, four real unknowns must be determined, precisely $H_{\mathrm{b}}, \vartheta_{b}, H_{0}$ and $\vartheta_{0}$. That problem can be solved by posing four independent equations, which descend

Fig. 5 Ampère's law represented by phasors 
Fig. 6 Equivalent parallel circuit of the field winding

from the application of the Carnot's theorem and the theorem of sines to the phasors triangle of Fig. 5. It follows

$H_{0}=\frac{N I}{d\left(1+K_{\mathrm{a}}\right)} \frac{1}{\sqrt{1+2 \frac{K}{\chi} \cos \Delta \vartheta+\left(\frac{K}{\chi}\right)^{2}}}$,

$\sin \vartheta_{0}=-\frac{K}{\chi} \frac{\sin (\Delta \vartheta)}{\sqrt{1+2 \frac{K}{\chi} \cos \Delta \vartheta+\left(\frac{K}{\chi}\right)^{2}}}$,

$H_{b}=\frac{K}{\chi} \frac{N I}{h} \frac{1}{\sqrt{1+2 \frac{K}{\chi} \cos \Delta \vartheta+\left(\frac{K}{\chi}\right)^{2}}}$,

$\sin \vartheta_{b}=\frac{\sin \Delta \vartheta}{\sqrt{1+2 \frac{K}{\chi} \cos \Delta \vartheta+\left(\frac{K}{\chi}\right)^{2}}}$,

where the relative magnetic length of the core, $K$, is

$K=\frac{h}{\mu_{\mathrm{r}} d+l}$.

For a given flux $\bar{\Phi}$, the reactive MMF due to the eddy currents is proportional to the core length $2 h$. In that sense, the core relative magnetic length $K$ represents the ratio between the magnetic potential drop produced by eddy currents reaction along the rotor path and the magnetic potential drop across the airgap and the armature. In other words, it expresses the relative intensity of the induced rotor reaction.

\subsection{Field winding lumped-circuit model: the magnetizing inductance}

One of the effects of the eddy currents is that to produce a phase shift between the impressed field current and the main flux crossing the airgap. This effect is already observed in Fig. 5, where $\bar{H}_{0}$ lags by the angle $\vartheta_{0}$ on the exciting MMF. With reference to the parallel lumped-circuit-model for the field winding represented in Fig. 6, the component of the field current $I$ sustaining the main flux $\bar{\Phi}$ and being in phase with it is

$I_{L}=I \cos \vartheta_{0}$.

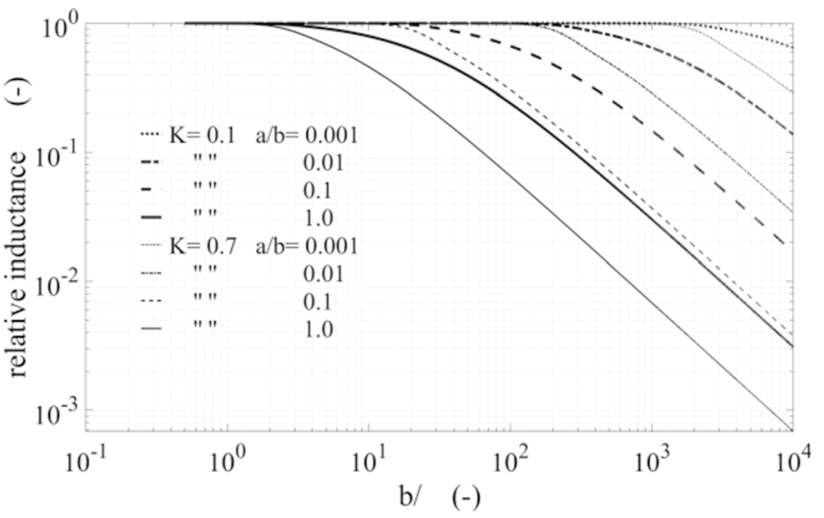

Fig. 7 Relative magnetizing inductance $\Lambda$

Therefore, $I_{L}$ appears in the following expression for the magnetizing inductance

$L_{\mathrm{m}}=\frac{\Psi}{I_{L}}=p \frac{2 N \Phi}{I \cos \vartheta_{0}}$,

where $\Psi$ is the magnetizing linked flux.

By substituting (26) in the modulus of (20), while considering (30), it results

$L_{\mathrm{m}}=L_{\mathrm{m}}(0) \Lambda$,

where $L_{\mathrm{m}}(0)$ is the null frequency or DC magnetizing inductance of the SM

$L_{\mathrm{m}}(0)=p \mu_{0} \frac{2 a b}{\left(d+\frac{l+h}{\mu_{\mathrm{r}}}\right)}(2 N)^{2}$,

and

$$
\Lambda=\frac{(1+K)}{\cos \vartheta_{0} \sqrt{1+2 \frac{K}{\chi} \cos \Delta \vartheta+\left(\frac{K}{\chi}\right)^{2}}}
$$

is the value of the AC-magnetizing inductance relative to the DC-one. Observing (21), (22) and (25) together with (33), it makes clear that $\Lambda$ is a function of the relative magnetic length of the core $K$, of the reduced thickness $b / \delta$ and of the pole cross-sectional shape ratio $a / b$. Figure 7 shows the dependency of $\Lambda$ on said parameters.

In Fig. 7, the eddy currents show a demagnetizing effect on the SM, which results in the drop of the magnetizing inductance when the reduced thickness increases. The same figure illustrates how the rotor geometric parameters govern this phenomenon. For the same core crosssectional shape (constant $a / b$ ) and the same frequency 


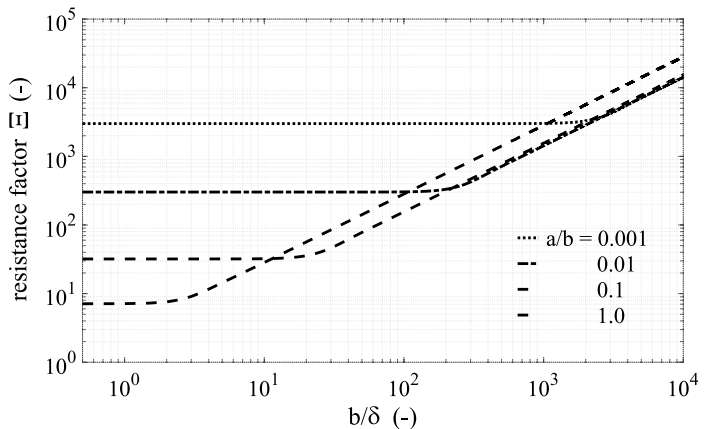

Fig. 8 Parametric representation of the resistance factor $\Xi$

(constant reduced thickness $b / \delta$ ), a higher degree of rotor reaction $K$ results in a lower magnetization inductance. In the same way, given a relative rotor core length (constant $K)$, the cross-sectional shape ratio $a / b$ sets the value of the reduced thickness $b / \delta$ (i.e., the frequency) at which the magnetizing inductance experiences the cut-off. It can be observed that the leaner the core cross-sectional shape, the higher the cut-off frequency for the field winding inductance.

\subsection{Field winding lumped-circuit model: the reflected resistance}

In Fig. 6, $R$ presents the reflected resistance according to Stoll [9], which relates to the eddy current loss. In the same circuit, it is possible to find out $R$ in dividing the winding self-induced voltage by the component of the excitation current in phase with it

$R=\frac{\omega \Psi}{I_{R}}=\frac{\omega p 2 N \Phi}{\left(-I \sin \vartheta_{0}\right)}$.

By substituting (29) in the modulus of (23), while considering (28) and (37), it results

$R=R\left(0^{+}\right) \cdot \Xi$

where

$R\left(0^{+}\right)=p \cdot 4 \rho \frac{(2 N)^{2}}{2 h}$

$R\left(0^{+}\right)$is a constant with the dimension of a resistance, which depends on the core length $2 h$, the iron resistivity $\rho$, the number of turns per pole-pair $2 N$ and the number of polepairs $p$. Since the reflected resistance cannot be sensed when the winding is supplied by a DC-voltage, said constant does not exist for null frequency. The resistance factor $\Xi$ represents instead a non-dimensional quantity
$\Xi=\frac{a}{b}\left(\frac{b}{\delta}\right)^{2} \frac{\chi}{\sin \Delta \vartheta}$

which takes into account the influence of the reduced thickness and the shape of the core cross section on the reflected resistance, as it is shown in Fig. 8.

In said figure, for a given core cross-sectional shape (constant $a / b$ ), a specific cut-off value for the reduced thickness $b / \delta$ can be recognized. It separates the range of constant reflected resistance from the range of increasing resistance. In particular, the leaner the core cross-sectional shape, the higher that cut-off value of the reduced thickness. Moreover, the constant resistance factor depends on the core cross-sectional shape alone before said cut-off, whereas, beyond it and for core profiles with $a / b<0.1$, the resistance factor shows a common asymptotic trend.

Given the not handy form of (37), $\Xi$ has been approximated by a simpler function $\tilde{\Xi}$ in the range of $a / b \in[0.001,1]$, with an overall error smaller than $2 \%$. The form of the approximating function is

$\tilde{\Xi}\left(\frac{b}{\delta}, \frac{a}{b}\right)=C_{R}\left(\frac{a}{b}\right) \sqrt{1+\left[\frac{\frac{b}{\delta}}{C_{C}\left(\frac{a}{b}\right)}\right]^{2}}$,

where

$C_{R}\left(\frac{a}{b}\right)=\frac{p_{R 2}\left(\frac{a}{b}\right)^{2}+p_{R 1}\left(\frac{a}{b}\right)+p_{R 0}}{\left(\frac{a}{b}\right)^{3}+q_{R 2}\left(\frac{a}{b}\right)^{2}+q_{R 1}\left(\frac{a}{b}\right)+q_{R 0}}$,

$C_{C}\left(\frac{a}{b}\right)=\frac{p_{C 2}\left(\frac{a}{b}\right)^{2}+p_{C 1}\left(\frac{a}{b}\right)+p_{C 0}}{\left(\frac{a}{b}\right)^{3}+q_{C 2}\left(\frac{a}{b}\right)^{2}+q_{C 1}\left(\frac{a}{b}\right)+q_{C 0}}$

with the following coefficients

$\left[\begin{array}{l}p_{R 2} \\ p_{R 1} \\ p_{R 0} \\ q_{R 2} \\ q_{R 1} \\ q_{R 0}\end{array}\right]=\left[\begin{array}{c}2.9274 \\ -2.6666 \\ 11.9760 \\ -3.2611 \\ 3.9813 \\ 0.0001\end{array}\right]$,

$\left[\begin{array}{l}p_{C 2} \\ p_{C 1} \\ p_{C 0} \\ q_{C 2} \\ q_{C 1} \\ q_{C 0}\end{array}\right]=\left[\begin{array}{c}9.5238 \\ -3.4514 \\ 12.7429 \\ 0.4677 \\ 6.0143 \\ -5.1124 \times 10^{-5}\end{array}\right]$ 
Fig. 9 Equivalent series circuit of the field winding

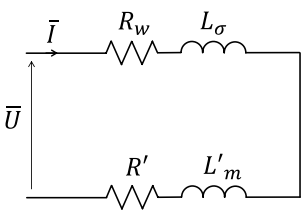

$C_{R}$ and $C_{C}$ used in (38) are rational functions of the shape ratio $a / b$ alone. They represent the resistance factor at low frequency and the cut-off value of the reduced thickness, respectively. With reference to Fig. $8, C_{R}$ scales the value of $R\left(0^{+}\right)$for setting its constant value at low frequency, as soon as the penetration depth largely exceeds the geometric average of the core half-dimensions $a$ and $b$

$\delta \gg \sqrt{a \cdot b} \rightarrow \frac{b}{\delta} \ll \sqrt{\frac{b}{a}}$.

The second function $C_{C}$ sets a limit for the relative value of the penetration depth, above which the reflected parallel resistance factor (37) is asymptotically proportional to the inverse of the penetration depth

$\frac{b}{\delta} \gg C_{C}\left(\frac{a}{b}\right) \rightarrow \Xi \cong \frac{C_{R}\left(\frac{a}{b}\right)}{C_{C}\left(\frac{a}{b}\right)} \frac{b}{\delta}$.

It is also possible to represent the lumped-circuit model of the field winding according to a series equivalent circuit as shown in Fig. 9. Said circuit is alternative to the one of Fig. 6.

Since both parallel and series equivalent lumped-circuit models must show the same input impedance, it must be true that

$L_{\mathrm{m}}^{\prime}=L_{\mathrm{m}} \frac{1}{1+(\omega \tau)^{2}}$

and

$R^{\prime}=R \frac{(\omega \tau)^{2}}{1+(\omega \tau)^{2}}$,

where

$\omega \tau=\frac{\omega L_{\mathrm{m}}}{R}=\frac{a}{b}\left(\frac{b}{\delta}\right)^{2} \frac{K}{K+1} \frac{\Lambda}{\Xi}$

descends from (31), (32),(35) and (36).

By substituting (47) and (35) in (46), the following expression for the series reflected resistance $R^{\prime}$ can be found

$R^{\prime}=R\left(0^{+}\right) \cdot \Xi^{\prime}$

where

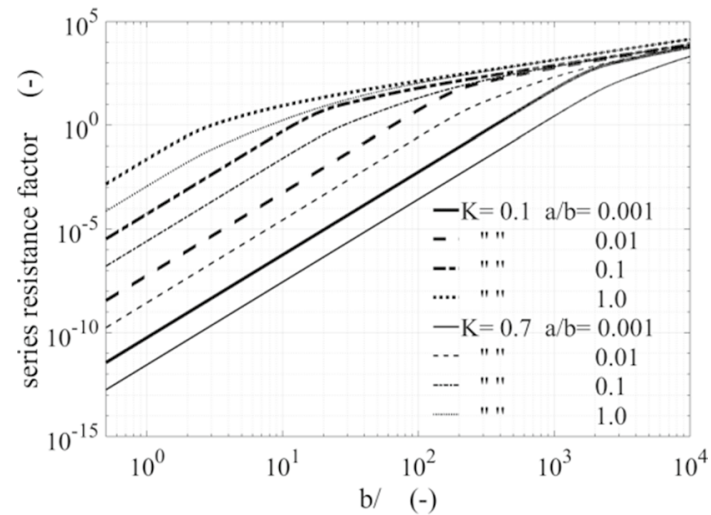

Fig. 10 Parametric representation of the series resistance factor $\Xi^{\prime}$

$\Xi^{\prime}=\Xi \frac{\left[\frac{a}{b}\left(\frac{b}{\delta}\right)^{2}\right]^{2}}{\left[\frac{a}{b}\left(\frac{b}{\delta}\right)^{2}\right]^{2}+\left(\frac{K+1}{K} \frac{\Lambda}{\Xi}\right)^{2}}$.

Equation (49) represents the non-dimensional factor for the series reflected resistance. It can be observed in (46) that the series and parallel reflected resistances converge asymptotically, as soon as the angular frequency is bigger than the inverse of the time constant $\tau$ (cut-off frequency). Equation (49) shows that the same convergence happens when the penetration depth becomes much smaller than the geometric average of $a$ and $b$. At low frequency instead, the series reflected resistance tends to zero, as it is predicted by (46). The described behavior is confirmed by the graphs shown in Fig. 10, where $\Xi^{\prime}$ is plotted versus $b / \delta$ for different values of the relative magnetic core length $K$ and of the cross-sectional shape ratio $a / b$.

In the previous Fig. 8, it can be observed that the multiplication factor $\Xi$ for the resistance of the parallel lumpedcircuit model does not depend on the parameter $K$, describing the relative magnetic length of the core. That means that it does not depend on the entity of the magnetic reaction of the armature and on the presence of the airgap, which are both summarized in $K$. In Fig. 10 instead, the multiplication factor $\Xi^{\prime}$ for the series lumped-circuit model shows its strong dependency on $K$. The parallel model has the advantage that, given an exciting MMF, as soon as an armature reaction reduces the main flux, it results in a corresponding drop of the induced electromotive force. This reduces in turn the eddy current losses without any need for adjusting the value of the parallel reflected resistance $R$. On the contrary, for the series equivalent lumped-circuit model of the rotor winding, once the MMF is constant in amplitude, a change in the eddy current losses due to the armature reaction always requires 


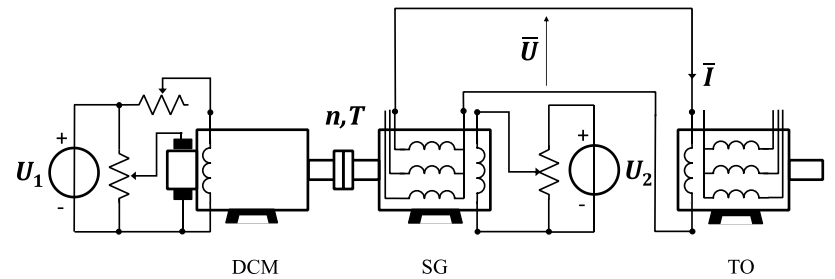

Fig. 11 Experimental setup

Table 1 Test objects main dimensions and characteristics

\begin{tabular}{llll}
\hline Symbol & Description & Machine 1 & Machine 2 \\
\hline$A$ & Rated power & $60 \mathrm{kVA}$ & $200 \mathrm{kVA}$ \\
$p$ & Pole pairs & 2 & 6 \\
$N$ & Turns per pole & 460 & 162 \\
$I_{n}$ & Rated field current & $10 \mathrm{~A}$ & $12.4 \mathrm{~A}$ \\
$2 a_{1}$ & Pole-shoe lamination thickness & - & $2.5 \mathrm{~mm}$ \\
$2 b_{1}$ & Pole-shoe width & - & $75 \mathrm{~mm}$ \\
$h_{1}$ & Pole-shoe-height & - & $45 \mathrm{~mm}$ \\
$2 a_{2}$ & Pole-core width & $70 \mathrm{~mm}$ & $75 \mathrm{~mm}$ \\
$2 b_{2}$ & Pole-core length & $200 \mathrm{~mm}$ & $303 \mathrm{~mm}$ \\
$2 h_{2}$ & Core-rim-core path length & $269 \mathrm{~mm}$ & $317 \mathrm{~mm}$ \\
$2 l$ & Armature pole pitch length & $322 \mathrm{~mm}$ & $226 \mathrm{~mm}$ \\
$2 e$ & Armature back iron radial thick- & $70 \mathrm{~mm}$ & $75 \mathrm{~mm}$ \\
& ness & & \\
$L_{\mathrm{m}}($ DC) & DC magnetizing inductance & $22 \mathrm{H}$ & $3.9 \mathrm{H}$ \\
$R_{\mathrm{w}}($ DC) & DC winding resist & $6.87 \Omega$ & $2.76 \Omega$ \\
\hline
\end{tabular}

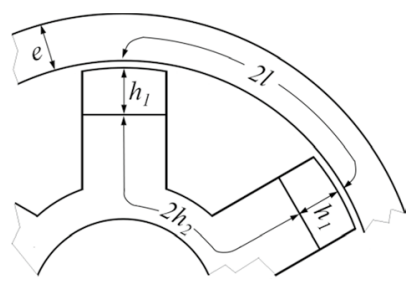

Fig. 12 The main dimensions of the magnetic circuit

an update of the series reflected resistance $R^{\prime}$, in order to give a correct account of the losses at constant current. This explains the dependency of $\Xi^{\prime}$ on $K$.

\subsection{Experimental setup}

In order to verify the validity of (35) in predicting the value for $R$ represented in Fig. 6, the field winding impedance of two different test-object (TO) synchronous machines has been measured over a given frequency range, by means of the setup depicted in Fig. 11.

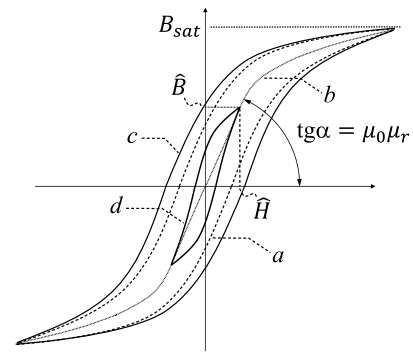

Fig. 13 Hysteresis losses estimation

A single phase of a synchronous generator SG supplies the field winding of the TO with a periodic voltage. The frequency $f$ of the applied voltage $\bar{U}$ is set by the rotational speed $n$ of a driving DC motor DCM. The amplitude of the voltage $\bar{U}$ is adjusted by controlling the field current of SG through the voltage divider applied to the generator $U_{2}$. In the following Table 1, and with reference to Fig. 12, the main features of the two TO are presented.

Said $\bar{U}=U \mathrm{e}^{\mathrm{j} \varphi_{U}}$ and $\bar{I}=I \mathrm{e}^{j \varphi_{I}}$ to be the fundamental harmonic components of voltage and current interesting the field winding, respectively, the related complex power $\bar{S}$ is equal to

$\bar{S}=\frac{1}{2} \bar{U} \cdot \bar{I}^{*}=\frac{U I}{2} \cos \left(\varphi_{U}-\varphi_{I}\right)+j \frac{U I}{2} \sin \left(\varphi_{U}-\varphi_{I}\right)$.

The real part of (50) accounts for the hysteresis, eddy current and excess losses in the TO, since the AC-resistance of the copper wire $R_{\mathrm{w}}$ [10] in Fig. 6 is negligible if compared with the reflected parallel resistance $R$. However, it is possible to prove that (50) represents the eddy current loss essentially, as soon as the amplitude of the applied AC voltage does not exceed the rated DC-voltage and as soon as it overcomes the cut-off frequency of the field winding. In fact, from the winding inductance and resistance enlisted in Table1, it is possible to determine the cutoff frequencies $0.05 \mathrm{~Hz}$ and $0.11 \mathrm{~Hz}$ for the field winding of the $60 \mathrm{kVA}$ and $200 \mathrm{kVA}$ rated SM, respectively. For an impressed $\mathrm{AC}$ voltage, having the amplitude of the DC-rated voltage and frequency $1 \mathrm{~Hz}$, the obtained excitation current amplitude must be smaller than one tenth of the rated DC-current for both TO. Moreover, the outbreak of the eddy current in the magnetic circuit counteracts the exciting MMF by reducing it even more. In that way, the general level of the flux density in the magnetic circuit of the TO becomes way lower than the DC-rated one. If the excitation winding was the primary winding of a transformer in the presence of few percent of the rated flux density, the active power being measured at the terminal of the winding itself would represent the copper loss of that transformer mainly, according to the well-known 
short-circuit test. In the SM field winding instead, the whirls of the eddy current in the rotor iron can be regarded as several secondary windings of the transformer closed in short circuit. Keeping the analogy, the fictitious "copper loss" measured at the field winding terminals is then the joule loss of a secondary winding made of iron, the iron of the rotor magnetic circuit. Under the condition of low flux in the core, the real part of (50) accounts for the eddy current loss essentially.

Nevertheless, an approximate estimation of the hysteresis and excess losses has been performed for testing the validity of this assumption. Figure 13 shows several hysteresis cycles insisting on the same magnetization curve $b$. The quasi-static hysteresis cycle $a$ encloses an area proportional to the hysteresis and the excess losses [11].

The wider hysteresis cycle $c$ encloses instead an area proportional to the total loss, so that its difference with the area enclosed by $a$ is proportional to the eddy current loss. If the value of the flux density is much smaller than the saturation one, the hysteresis and the excess losses are represented by the area of a smaller symmetrical hysteresis cycle $d$, delimited by the extremal points $(-\hat{H},-\hat{B})$ and $(\hat{H}, \hat{B})$ in Fig. 13. Since the rotors of synchronous machines, in massive or partially laminated execution, consist of soft magnetic materials made of low-carbon iron or iron-alloys, the ratio between $\hat{B}$ and $\hat{H}$ is characterized by a pretty high value of the relative permeability, typically $\mu_{\mathrm{r}}>2000$ [12]. Imagining to know $\hat{B}$, the power $P_{\text {he }}$ related to the hysteresis and excess losses can be overestimated by $\tilde{P}$

$P_{\text {he }}<\tilde{P}=p 2 \frac{\hat{B}^{2}}{\mu_{\mathrm{r}}^{*} \mu_{0}} V_{\text {core }}$,

where $V_{\text {core }}$ stands for the core volume and $\mu_{\mathrm{r}}^{*}$ is a constant relative permeability, higher than 2000 . By neglecting the influence of the field winding stray inductance, the peak value of the average flux density in the core can be estimated by

$\hat{B} \cong \frac{U / p}{\omega 2 N} \frac{1}{4 a b}$.

Equation (51) presents the limitation to neglect the flux density distribution in the core cross section. For this reason, its result has been compared with the more consolidated method of the complex permeability [13]. In this last method, the flux density lags the magnetic field on the angle $\theta$ according to

$\bar{B} \cong \mu_{\mathrm{r}}(\|\bar{B}\|) \mu_{0} \mathrm{e}^{-j \theta} \bar{H}$.

Assumed the core made of a soft magnetic materialsuch as, e.g., low-carbon steel 1006-for the $60 \mathrm{kVA} \mathrm{SM}$

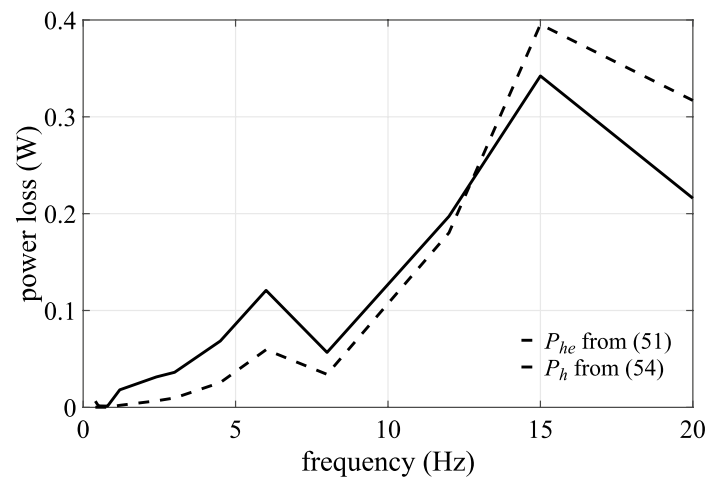

Fig. 14 Comparison between results from (51) and (54) in the 60 kVA TO

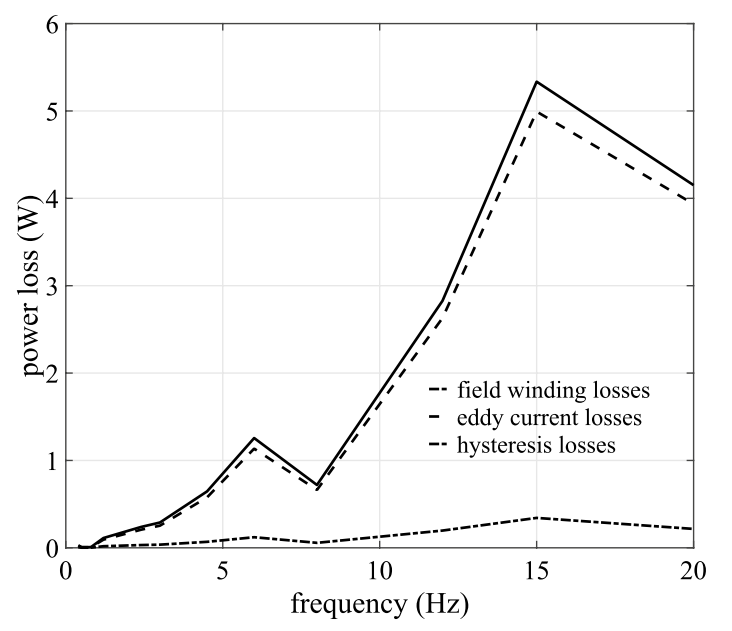

Fig. 15 Losses repartition in the $60 \mathrm{kVA}$ TO during measurements

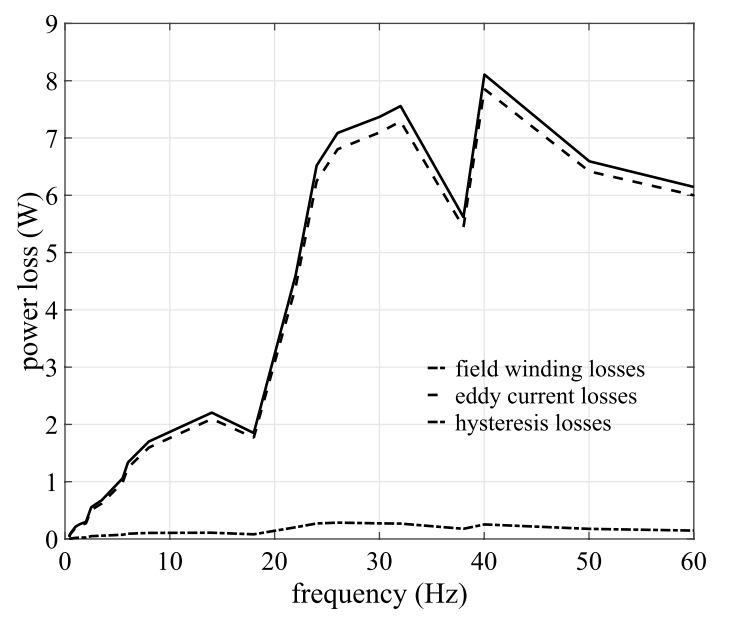

Fig. 16 Losses repartition in the $200 \mathrm{kVA}$ TO during measurements 


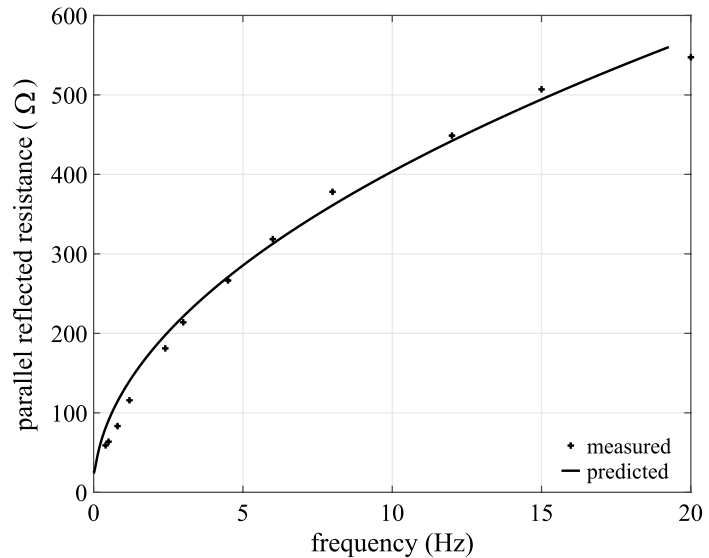

Fig. 17 Eddy currents related parallel resistance for $60 \mathrm{kVA}$ TO

Fig. 18 Eddy currents related parallel resistances for the 200 kVA TO

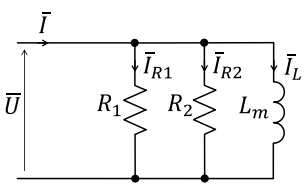

and performed a stationary 2D-FEM analysis with $\theta=8^{\circ}$, the hysteresis loss can be estimated by

$P_{h} \cong\left(1+\frac{a}{b}\right) b 2 h f \int_{-a}^{a} \pi\|\bar{B}\|\|\bar{H}\| \sin \theta \mathrm{d} x$

where $\left(1+\frac{a}{b}\right)$ represents the $2 \mathrm{D}$-to-3D correction factor.

In Fig. 14, it can be observed that (51) deviates significantly from (54), but it catches the same order of magnitude for the loss.

\section{Results}

In Figs. 15 and 16, the measured active power absorbed by the field winding in the $60 \mathrm{kVA}$ and $200 \mathrm{kVA}$ test machine, respectively, has been split between eddy current- and hysteresis + excess losses by means of (50) and (51).

Since both voltages $U_{1}$ and $U_{2}$ in the setup of Fig. 11 have been adjusted during the measurements, in order to target the wanted frequency, the curves of Figs. 15 and 16 do not represent values obtained at constant excitation flux. This explains why the curves are broken and not smooth. Both figures show that the aggregated hysteresis- and excess losses are way smaller than the eddy current loss, confirming by that the prevision done in the previous paragraph. The aggregated hysteresis- and excess losses, which accounts for less than $12 \%$ in both cases, could have been neglected in the determination of the eddy currents-related AC-resistance

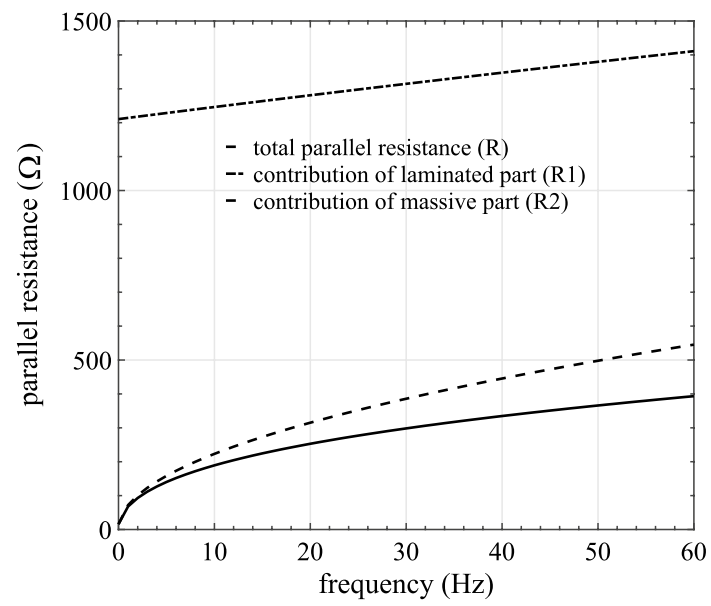

Fig. 19 Eddy currents related parallel resistances for the $200 \mathrm{kVA}$ TO

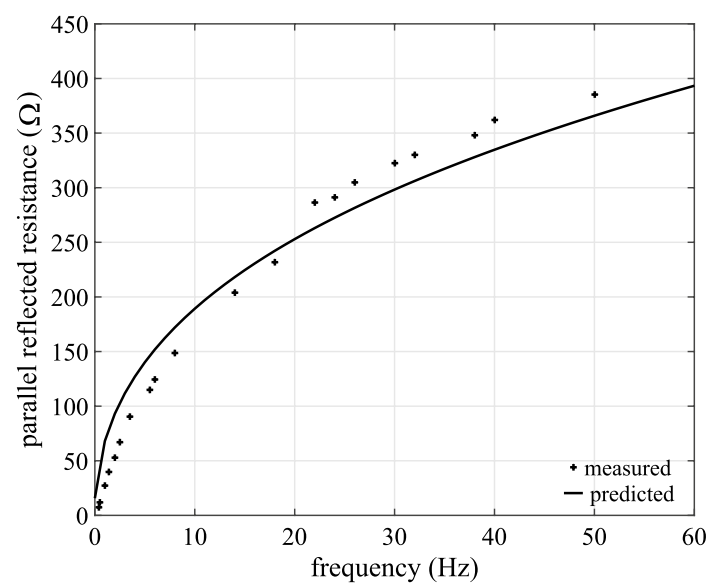

Fig. 20 Eddy currents related parallel resistance for the $200 \mathrm{kVA}$ TO

$R_{\mathrm{e}}=\frac{(\operatorname{Re}[\bar{S}]-\tilde{P})}{U^{2}}$,

without introducing a relevant error in the indirectly measured reflected resistance.

Figure 17 shows the comparison between said resistance $R_{\mathrm{e}}$ and the predicted one calculated by (35) for the $60 \mathrm{kVA}$ rated machine. A specific rotor iron resistivity $\rho=90 \mathrm{n} \Omega \mathrm{m}$ and an average relative permeability $\mu_{\mathrm{r}}=1000$ have been required for fitting the values of $R_{\mathrm{e}}$.

The rotor of the $200 \mathrm{kVA}$ machine has laminated poleshoes which extend for a radial height $h_{1}$ and a massive pole core and rotor rim showing a whole length $2 h_{2}$ as represented in Table 1 and Fig. 12. In that case, since the two portions of the rotor magnetic circuit are crossed by the same flux, the reflected resistances related to each portion must share the same electromotive force. Therefore, they can be represented by two resistances in parallel as shown in Fig. 18. R1 


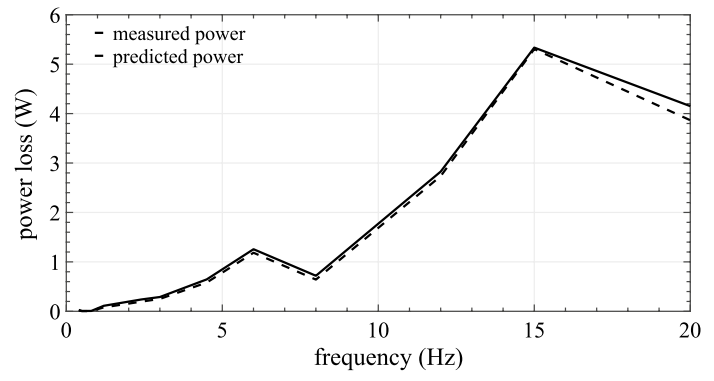

Fig. 21 Comparison measured and predicted power for the $60 \mathrm{kVA}$ TO

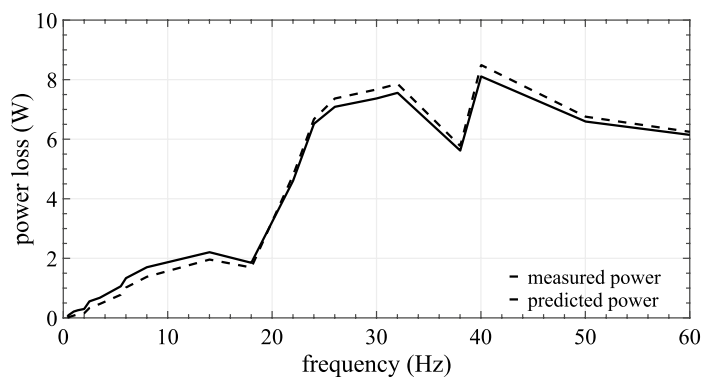

Fig. 22 Comparison between measured and predicted power for the 200 kVA TO

is related to the laminated pole-shoe and $\mathrm{R} 2$ to the massive pole core and rim. Both have been calculated by (35).

Correspondingly, Fig. 19 shows the contributions of each rotor portion, pole-shoes and pole core + rim, to the total reflected resistance, in function of the applied voltage frequency.

The laminated part (pole-shoes), which is seat of less eddy current loss in comparison with the remaining part of the rotor, shows the highest value of the reflected resistance $\left(R_{1}>R_{2}\right)$.

In Fig. 20, the fit of (35) to the $R_{e}$ for the $200 \mathrm{kVA}$ machine has required a specific rim-pole steel resistivity $\rho=140 \mathrm{n} \Omega \mathrm{m}$, a specific pole-shoe steel resistivity $\rho=470 \mathrm{n} \Omega \mathrm{m}$ and an average relative permeability $\mu_{\mathrm{r}}=1000$.

Figures 17 and 20 prove that it is possible to estimate the reflected resistance of a salient pole synchronous machine field winding by the analytical approach presented in the method of this paper. Even though the power supplied to the field winding during the measurements shows a large variability in Figs. 15 and 16, the relative plots of the measured reflected resistances in Figs. 17 and 20 are both very smooth and present a clear trend versus the frequency. This is mainly due to the fact that said resistance is independent of the voltage level applied to the rotor circuit. This favorable circumstance makes it possible to use the suggested model for obtaining a rough estimate of the eddy current loss induced in the rotor, as soon as the control voltage $U$ is known. Once the reflected resistance in the model of Fig. 6 has been guessed by (35), the estimated eddy current loss can be predicted by,

$P_{\mathrm{e}} \cong \frac{U^{2}(f)}{2 R(f)}$.

Figures 21 and 22 show the comparison between the active power measured on both $\mathrm{TO}$ and the predicted value of the eddy current loss (59), comparison made possible by the small weight of the hysteresis and excess losses on the total loss documented above.

The control voltage $U$ used in (56) is the one recorded during the measurements performed on the two synchronous machines, whereas the resistance $R$ is the one used as fit in Figs. 17 and 20, respectively. Both Figs. 21 and 22 present a trend of the predicted power, which follows pretty well the evolutions of the measured power. This result proves that the modeled reflected resistance (35) catches the effects of both independent variables on the eddy current generation, the applied voltage level $U$ and the frequency $f$. By doing so, it supports the fundamental idea of this work, that to characterize the losses induced by the excitation control by means of a frequency-dependent resistance, the value of which is related to the SM main rotor parameters. In fact, from Figs. 6 and 8, it can be seen that for $\delta \gg \sqrt{a \cdot b}$ (i.e., rotor iron fully penetrated by the magnetic field) the magnetizing inductance and the reflected resistance are substantially unaffected by the eddy currents. The first one equals its DC value, and the second one remains constant and independent from the frequency. As soon as $\delta \ll \sqrt{a \cdot b}$, the magnetizing inductance starts to decrease proportionally to the penetration depth and the reflected resistance increases instead inversely proportional to that. This allows to draw some conclusions about the dependency of the eddy current loss on the frequency, when the exciting MMF is held constant. Considering (11) and the just above-mentioned remarks, it can be found that

$P_{\mathrm{e}}=\frac{1}{2} \frac{\left[\omega L_{\mathrm{m}}(0) I\right]^{2}}{R_{0}} \propto f^{2} \quad$ for $\delta \gg \sqrt{a \cdot b}$

and

$P_{\mathrm{e}}=\frac{1}{2} \frac{\left(\omega L_{\mathrm{m}} I\right)^{2}}{R} \propto \sqrt{f}$ for $\delta \ll \sqrt{a \cdot b}$.

In particular, for the case examined in (57), as soon as $a / b<0.1$, it can be found from (39) that.

$C_{R}\left(\frac{a}{b}\right) \cong 3 \frac{b}{a}$. 


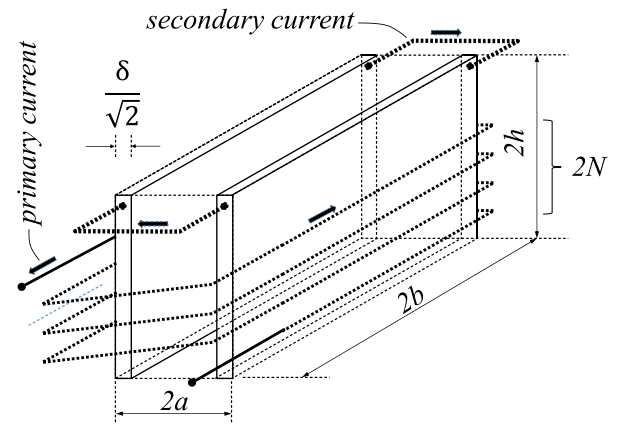

Fig. 23 Transformer-like model for the eddy current account

Taking into account (36), (38) and (59), the eddy current loss can be then expressed by

$P_{\mathrm{e}}=p \frac{1}{2} \frac{(\omega 2 N B 4 a b)^{2}}{R\left(0^{+}\right) \cdot C_{R}\left(\frac{a}{b}\right)}=\frac{\pi^{2} f^{2} B^{2}(2 a)^{2}}{6 \rho} \cdot V_{\text {core }}$

which returns the classical analytical result (1) for the eddy current loss in a fully penetrated thin metal sheet of thickness $d_{l}=2 a$. In the case considered in (58) instead, as soon as $a / b<0.1$, it can be easily recognized from Fig. 8 that

$\Xi\left(\frac{b}{\delta}\right) \cong \sqrt{2} \frac{b}{\delta}$.

A first simple estimate of the reflected resistance is then possible by using (36), (38) and (61)

$R=p 4 \rho \frac{(2 N)^{2}}{2 h} \frac{\sqrt{2} b}{\delta}=p \rho \frac{2 \cdot 2 b}{2 h \cdot \frac{\delta}{\sqrt{2}}}(2 N)^{2}$.

The reflected resistance (62) found by this way can be interpreted as the secondary resistance of a transformer with voltage transformation ratio $2 N: 1$, transferred to its primary winding and taken into account $p$-times, as Fig. 23 shows. The resistance of the secondary winding single turn is that of a conductive layer presenting resistivity $\rho$, length 2 times $2 b$ and cross-sectional area equal to $2 h$ times $\delta / \sqrt{2}$.

This result shows that the induced eddy currents can be regarded as concentrated underneath the lateral surface of the not fully penetrated thin metal sheet, within a thickness of ca. $70 \%$ of the penetration depth.

Besides the encouraging results obtained by the analytical approach used in the present work, it should be noted that both predicted resistances in Figs. 17 and 20 underestimate slightly the real losses at low frequency. This is the consequence of having assumed an average constant magnetic permeability for the magnetic circuit all over the examined frequency range. Hariharan [14] has shown that the iron core flux density increases from the middle of the iron core toward its lateral surface, as soon as the eddy currents concentrate toward the lateral boundary of the pole and rotor rim. This means that, when the rotor pole is fully penetrated, the magnetizing inductance should be generally bigger than the one calculated by the simplifying hypothesis assumed in here. Therefore, for the same MMF a higher magnetic flux should be expected, which would be responsible for a higher loss than the here predicted one. On the other hand, it must be noticed that the used simplistic hypothesis makes the model and its outcomes easy to be derived and handled. Finally, having neglected the stray inductance of the winding in the suggested model implies that the highest frequency limit for model validity is presumably that, which makes the magnetizing inductance of the winding one order of magnitude larger than the stray inductance.

\section{Conclusion}

A parallel and a series equivalent lumped-circuit model for the field winding of salient pole synchronous machines has been obtained by means of a simple analytic electromagnetic approach. It manages to interpret and to reproduce many effects, which are related to the outbreak of eddy currents in the rotor of a salient pole synchronous machine, such as the main flux lagging on the exciting MMF, the weakening of the field winding inductance and the increase in the winding AC-resistance. In particular, the achieved model is able to relate the eddy current magnetic reaction and loss to the main geometrical and physical properties of the rotor winding. Said model has been tested on two salient pole synchronous machines by comparing its predictions in terms of AC-resistance and induced eddy current loss, with the evidences of measurements performed on their field windings. In spite of the simplifying assumptions behind its achievement, the model is able to point out to the essential factors influencing the generation of eddy current in the rotor and to perform a rough estimate of its loss. It can be useful in the design of a SM for verifying the suitability of a given rotor arrangement for the needed control strategy, as well as, in the analysis of those, sometimes older, existing machines, where the material properties and the exact geometry of the rotor are unknown. Finally, in expressing the dependency of the field winding inductance and resistance on the frequency, the model can provide an operational formulation of the excitation winding, which better suits the dynamic analysis involving voltage/stability control loops.

Acknowledgements Open access funding provided by Uppsala University. This research has been carried out within the HydroFlex project, which has received funding from the European Union Horizon 2020 research and innovation programme under Grant Agreement No 764011 . 
Open Access This article is licensed under a Creative Commons Attribution 4.0 International License, which permits use, sharing, adaptation, distribution and reproduction in any medium or format, as long as you give appropriate credit to the original author(s) and the source, provide a link to the Creative Commons licence, and indicate if changes were made. The images or other third party material in this article are included in the article's Creative Commons licence, unless indicated otherwise in a credit line to the material. If material is not included in the article's Creative Commons licence and your intended use is not permitted by statutory regulation or exceeds the permitted use, you will need to obtain permission directly from the copyright holder. To view a copy of this licence, visit http://creativecommons.org/licenses/by/4.0/.

\section{References}

1. Kriezis EE, Tsiboukis TD et al (1992) Eddy currents: theory and applications. Proc IEEE. https://doi.org/10.1109/5.168666

2. Nagel JR (2018) Induced eddy currents in simple conductive geometries: mathematical formalism describes the excitation of electrical eddy currents in a time-varying magnetic field. IEEE Antennas Propag Mag. https://doi.org/10.1109/MAP.2017.27742 06

3. Pyrhonen J, Jokinen T et al (2008) Design of rotating electrical machines. Wiley, New York

4. Pérez-Loya JJ, Abrahamsson CJD et al (2017) Demonstration of active compensation of unbalanced magnetic pull in synchronous machines. Cigre Sci Eng. http://www.diva-portal.org/smash/recor d.jsf?pid=diva2\%3A1133821\&dswid $=-5656$

5. Pérez-Loya JJ, Abrahamsson CJD et al (2018) Demonstration of synchronous motor start by rotor polarity inversion. IEEE Trans Ind Electron. https://doi.org/10.1109/TIE.2017.2784342
6. Dreyfus L (1915) Die Feldverteilung und Wirbelströmbildung in den Ankern von Dynamomaschinen bei Unmagnetisierung durch hochperiodige Wechsel- und Drehfelder. Archiv für Elektrotechink. https://doi.org/10.1007/BF01660158

7. Nethe A (1991) Einschaltströme in Spulen mit leitendem permeablem Kern bei Berücksichtigung der induzierten Wirbelströme. Archiv für Elektrotechnik. https://doi.org/10.1007/BF01577460

8. Jäschke C, Schegner P (2015) Analytic computation of magnetizing inductance of current instrument transformers under consideration of eddy currents. IEEE Trans Magn. https://doi.org/10.1109/ TMAG.2015.2438320

9. Stoll RL (1974) Analysis of eddy currents. Clarendon Press, Oxford, $\mathrm{p} 15$

10. Pyrhonen J, Jokinen T et al (2008) Design of rotating electrical machines. Wiley, New York, pp 262-265

11. Bertotti G (1998) Hysteresis in magnetism. Academic Press, San Diego, p 402

12. Fiorillo F (2004) Measurements and characterization of magnetic materials. Academic Press, San Diego, p 33

13. Stoll RL (1974) Analysis of eddy currents. Clarendon Press, Oxford, $p 16$

14. Hariharan V (2019) The effect of eddy currents on the magnetic inductance of synchronous machines with large poles. Dissertation, Uppsala University. http://uu.diva-portal.org/smash/recor d.jsf?pid=diva2\%3A1330306\&dswid=-2075

Publisher's Note Springer Nature remains neutral with regard to jurisdictional claims in published maps and institutional affiliations. 